\title{
Novel Transmission Schemes for Multicell Downlink MC/DS-CDMA Systems Employing Time- and Frequency-Domain Spreading
}

\author{
Jia Shi and Lie-Liang Yang \\ School of ECS, University of Southampton, SO17 1BJ, United Kingdom \\ Tel: 0044-(0)23-8059 3364, Email: js22g09,1ly@ecs.soton.ac.uk, http://www-mobile.ecs.soton.ac.uk
}

\begin{abstract}
This contribution investigates the achievable error performance of transmitter preprocessing in the downlink multicell multicarrier direct-sequence code-division multiple-access (MMC/DS-CDMA) systems employing both time (T)-domain and frequency (F)-domain spreading. Three types of multiuser transmitter preprocessing (MUTP) schemes are studied and compared, when assuming communications over frequencyselective Rayleigh fading channels. The first one is a single-cell minimum mean-square error MUTP (SMMSE-MUTP1), which only aims at suppressing the intracell interference (IntraCI). The second one is also a single-cell MMSE-MUTP (SMMSE-MUTP2), which tries to suppress both the IntraCI and intercell interference (InterCI). The final one is the multicell cooperative MMSE-MUTP (CMMSE-MUTP), which exploits the multicell diversity (or macro-diversity) for interference suppression and performance enhancement. Furthermore, power-allocation in these schemes is considered. Our studies show that the CMMSE-MUTP is capable of achieving the best error performance among the three schemes considered. However, it demands an extremely high complexity, as it requires information exchange among the base-stations (BSs) with the aid of a backhaul system. By contrast, when the number of mobile terminals (MTs) supported by each cell is not very high, the SMMSE-MUTP2, which does not require any intercell cooperation, can effectively mitigate both the IntraCI and the InterCI.
\end{abstract}

\section{INTRODUCTION}

In the future generations of cellular communication systems, intracell interference (IntraCI) and intercell interference (InterCI) are two highly challenging issues, as they limit both the achievable capacity and the achievable reliability. In multicell downlink communications, well designed base-station (BS) preprocessing schemes are capable of effectively mitigating the downlink interferences. Furthermore, if BS cooperation is available, the spatial diversity (or so-called macrodiversity) provided by the different BSs' antennas may be used for further improving the capacity and/or reliability the multicell systems.

Transmitter preprocessing for the single-cell downlink scenarios has been widely investigated, such as, in [1-4], based on different objectives of optimization, when perfect channel state information (CSI) is assumed. For example, in [1], the preprocessing vector has been optimized jointly with the power-allocation under the individual signal to interference-plus-noise ratio (SINR) constraints, when either the reciprocal uplink/downlink systems or the non-reciprocal uplink/downlink systems are considered. By contrast, in [2], an iterative algorithm has been proposed, which also jointly optimize the preprocessing vector and the power-allocation. In the context of the multicell systems, transmitter preprocessing optimization has been considered in [5-9], when assuming that the invoked BSs ideally share their data and CSI. In [8], multicell preprocessing schemes have been designed based on maximizing the signal-to-interference leakage-plusnoise ratio (SILNR), in order to reduce the amount of data need to be exchanged via the backhaul system. Subject to the quality of service (QoS) constraints of individual mobile terminals (MTs), multicell downlink transmission motivating to minimizing the total downlink transmit power has been studied in [9], when imperfect CSI is assumed at both the transmitter and the receiver sides. As illustrated in [10, 11], the coordinated multicell transmission is able to fully remove the InterCI, when ideal data and CSI exchange among BSs is assumed.
However, the required huge information exchange among BSs as well as the daunting channel estimation demanded are highly challenging for implementation of these schemes in practice. Hence, for the sake of complexity reduction, in [12], the authors have investigated an adaptive InterCI cancellation downlink transmission scheme for the multicell systems. Under this scheme, the single-cell assisted preprocessing is employed by the BS when the edge SNR is below the threshold, while the coordinated multicell preprocessing is operated when the edge SNR is above the required threshold.

In this contribution, we study the BS preprocessing schemes for the downlink multicell multicarrier direct spread code division multiple access (MMC/DS-CDMA) systems employing both time (T)-domain and frequency (F)-domain spreading [13]. We consider specifically the MMC/DS-CDMA, as it has a range of advantages, including high capacity and flexibility, less severe peak-to-average power ratio (PAPR) problem, relatively low-chip-rate and short spreading codes to achieve low-rate signal processing, etc. [13-15]. Specifically, according to [17], where single-cell scenarios are considered, linear multiuser transmitter preprocessing (MUTP) schemes can be designed from their counterpart uplink multiuser detection (MUD) schemes, based on the equivalency relationship between the uplink and downlink channels. In this paper, we extend the principles to the multicell scenarios and design the minimum mean-square error (MMSE) MUTP with InterCI suppression, which is referred to as the SMMSE-MUTP2. In order to illustrate its advantage, we also derive and study the MMSE-MUTP without InterCI suppression, which is referred to as SMMSE-MUTP1. Furthermore, motivating to show the upper-bound performance of the multicell systems employing MMSE-assisted preprocessing, the MMSE-MUTP with full BS cooperation supported by ideal data and CSI exchange among BSs is investigated. This scheme is referred to as the CMMSE-MUTP. Additionally, for the above-mentioned MMSEMUTP schemes, different power-allocation (normalization) methods are considered and compared. Finally, the BER performance of the MMC/DS-CDMA systems with various MUTP and power-allocation schemes is investigated, when assuming communications over frequency selective Rayleigh fading channels. Our studies and performance results show that the CMMSE-MUTP is capable of achieving the best error performance among the three schemes considered, while demanding the extremely high complexity. When the number of MTs supported by each cell is not very high, the SMMSE-MUTP2, which is designed without requiring any intercell information exchange, can effectively mitigate both the IntraCI and the InterCI.

The rest of the paper is organized as follows. Section II provides the system model and states the main assumptions. In Section III, we derive the three MUTP schemes. Section IV demonstrates the performance results and provides the related discussion. Finally, Section V summarizes the conclusions.

\section{System Models}

The considered downlink multicell MC DS-CDMA (MMC/DSCDMA) system employing TF-spreading has the structure as shown in 


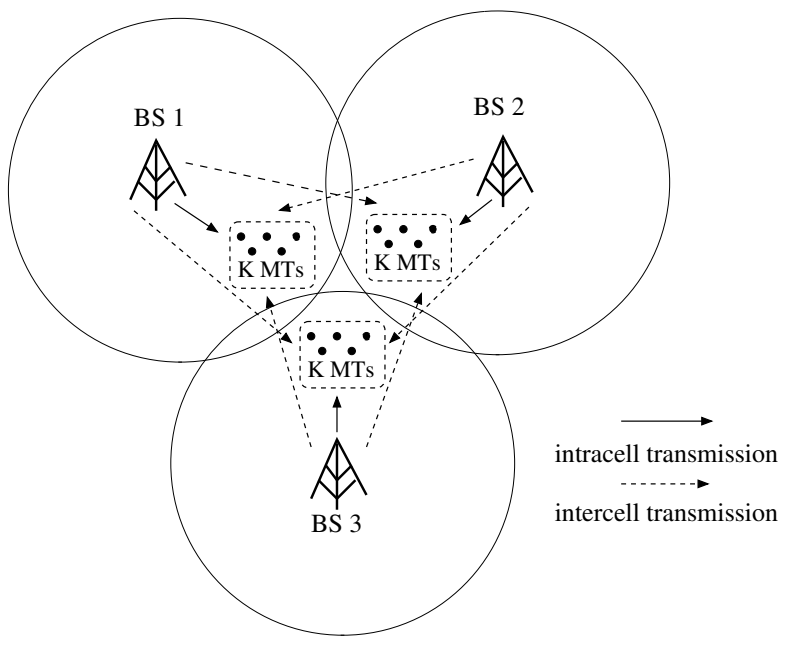

Fig. 1. Conceptual structure for the downlink MMC/DS-CDMA Systems.

Fig. 1. For the purpose of study and also for the sake of simplicity, we assume that the system consists of three cells, each of which has one BS supporting $K$ MTs. We assume that each of the communication terminals, including the BSs and MTs, employs one antenna for receiving and transmitting signals. The $K$ MTs in one cell are assumed to have the similar distance to their own BS as well as the similar distance to the two neighbor cells. Signals transmitted from BSs to MTs are MC/DS-CDMA signals supported by TF-spreading [13], the T- and F-domain spreading factors are expressed as $N_{t}$ and $N_{f}$, respectively.

We assume that the uplink and downlink use time-division duplex (TDD) and that an uplink channel and its corresponding downlink channel are reciprocal. Hence, the CSI of a downlink channel can be derived by estimating its reciprocal uplink channel. The channels between BSs and MTs are assumed to experience frequency-selective Rayleigh fading. However, owing to using multiple subcarriers, we assume that each subcarrier experiences independent flat Rayleigh fading. In this contribution, our MUTP is optimized in the sense of minimum mean-square error (MMSE).

Based on the above assumptions, the signaling for the MMC/DSCDMA system can be described as follows. Let us assume that the symbols transmitted by the $m$ th, $m=1,2,3$, BS to its $K$ MTs are expressed as $\boldsymbol{x}_{m}=\left[x_{m, 1}, x_{m, 2}, \ldots, x_{m, K}\right]^{T}$, where $x_{m, k}$ is assumed to satisfy $E\left[x_{m, k}\right]=0$ and $E\left[\left|x_{m, k}\right|^{2}\right]=1$. Then, after stacking the observations obtained by the $K$ MTs in Cell $i$, where each MT obtains $N_{t} N_{f}$ observations corresponding to the $N_{f}$ subcarriers and $N_{t}$ timechips, into a vector $\boldsymbol{y}_{i}$, we can show that $\boldsymbol{y}_{i}$ is

$$
\begin{aligned}
\boldsymbol{y}_{i} & =\sum_{m=1}^{3} \sum_{n=1}^{3} \boldsymbol{A}_{i m} \overline{\boldsymbol{P}}_{m}^{(n)} \boldsymbol{x}_{m}+\boldsymbol{n}_{i} \\
& =\boldsymbol{A}_{i} \overline{\boldsymbol{P}} \boldsymbol{x}+\boldsymbol{n}_{i}, i=1,2,3
\end{aligned}
$$

where $\boldsymbol{x}=\left[\boldsymbol{x}_{1}^{T}, \boldsymbol{x}_{2}^{T}, \boldsymbol{x}_{3}^{T}\right]^{T}, \boldsymbol{y}_{i}=\left[\boldsymbol{y}_{i, 1}^{T}, \ldots, \boldsymbol{y}_{i, K}^{T}\right]^{T}$ is a $N_{t} N_{f} K$ length vector with $\boldsymbol{y}_{i, k}$ an $N_{t} N_{f}$-length vector containing the observations obtained by the $k$ th MT in Cell $i$. In (1), $\boldsymbol{A}_{i}=\left[\boldsymbol{A}_{i 1}, \boldsymbol{A}_{i 2}, \boldsymbol{A}_{i 3}\right]$ is a $\left(N_{t} N_{f} K \times 3 N_{t} N_{f}\right)$ matrix, where $\boldsymbol{A}_{i m}$ is structured by the channels between the $m$ th BS and the $K$ MTs of Cell $i$. In detail, $\boldsymbol{A}_{i m}=\left[\boldsymbol{A}_{\tilde{i m}, 1}, \ldots, \boldsymbol{A}_{i m, K}\right]^{T}$ is a $\left(N_{t} N_{f} K \times N_{t} N_{f}\right)$ matrix with $\boldsymbol{A}_{i m, k}=\tilde{\boldsymbol{A}}_{i m, k} \otimes \boldsymbol{I}_{N_{t}}$, where $\otimes$ represents the Kronecker product, $\boldsymbol{I}_{N_{t}}$ is a $\left(N_{t} \times N_{t}\right)$ identity matrix, while $\tilde{\boldsymbol{A}}_{i m, k}=\alpha_{i m, k} \times$ $\operatorname{diag}\left\{a_{i m, k}^{(1)}, \ldots, a_{i m, k}^{\left(N_{f}\right)}\right\}$ is a $\left(N_{f} \times N_{f}\right)$ diagonal matrix formed by the channel gains of the $N_{f}$ subcarriers with respect to the $m$ th BS and the $k$ th MT in the cell $i$. We assume that $\left|a_{i m, k}^{\left(n_{f}\right)}\right|, n_{f}=1, \ldots, N_{f}$, obeys the Rayleigh distribution with $E\left[\left|a_{i m, k}^{\left(n_{f}\right)}\right|^{2}\right]=1$, while $\alpha_{i m, k}$ characterizes the pathloss of the communication link from the $m$ th BS to the $k$ th MT in Cell $i$. For the sake of simplicity, we assume that $\alpha_{i m, k}=1$, when intracell transmission is considered, i.e., when $i=m$, while $0 \leq \alpha_{i m, k} \leq 1$ is a uniformly distributed random variable for the intercell interfering signals corresponding to $i \neq m$. In (1), the preprocessing matrix $\overline{\boldsymbol{P}}$ is a $\left(3 N_{t} N_{f} \times 3 K\right)$ matrix, which is in the form of

$$
\overline{\boldsymbol{P}}=\left[\begin{array}{ccc}
\overline{\boldsymbol{P}}_{1}^{(1)} & \overline{\boldsymbol{P}}_{2}^{(1)} & \overline{\boldsymbol{P}}_{3}^{(1)} \\
\overline{\boldsymbol{P}}_{1}^{(2)} & \overline{\boldsymbol{P}}_{2}^{(2)} & \overline{\boldsymbol{P}}_{3}^{(2)} \\
\overline{\boldsymbol{P}}_{1}^{(3)} & \overline{\boldsymbol{P}}_{2}^{(3)} & \overline{\boldsymbol{P}}_{3}^{(3)}
\end{array}\right]
$$

where $\overline{\boldsymbol{P}}_{m}^{(n)}=\left[\overline{\boldsymbol{p}}_{m, 1}^{(n)}, \ldots, \overline{\boldsymbol{p}}_{m, K}^{(n)}\right]$ is a $\left(N_{t} N_{f} \times K\right)$ preprocessing matrix calculated by the $n$th BS for the $K$ MTs in Cell $m$, with $\overline{\boldsymbol{p}}_{m, k}^{(n)}$ the $N_{t} N_{f}$-length preprocessing vector for the $k$ th MT in Cell $m$. At last, in (1), $\boldsymbol{n}_{i}=\left[\boldsymbol{n}_{i, 1}^{T}, \ldots, \boldsymbol{n}_{i, K}^{T}\right]^{T}$ is a $N_{t} N_{f} K$-length vector, where $\boldsymbol{n}_{i, k}=\left[n_{i, k}^{(1)}, \ldots, n_{i, k}^{\left(N_{t} N_{f}\right)}\right]^{T}$ is a $N_{t} N_{f}$-length vector corresponding to the $k$ th MT in Cell $i$. Each element of $\boldsymbol{n}_{i}$ is assumed to obey the complex Gaussian distribution with zero mean and a variance of $2 \sigma_{d}^{2}$, where $\sigma_{d}^{2}=1 /\left(2 \bar{\gamma}_{s}\right)$ with $\bar{\gamma}_{s}$ denoting the average signal-to-noise ratio (SNR) per symbol.

In this contribution, three types of MUTP schemes are investigated and compared, which are the: 1) SMMSE-MUTP1 - singlecell MMSE-MUTP without InterCI suppression; 2) SMMSE-MUTP2 - single-cell MMSE-MUTP with InterCI suppression; 3) CMMSEMUTP - multi-cell cooperative MMSE-MUTP. Note that, when the single-cell based preprocessing, either the SMMSE-MUTP1 or SMMSE-MUTP2, is employed, the preprocessing matrix of (2) becomes $\overline{\boldsymbol{P}}=\operatorname{diag}\left\{\overline{\boldsymbol{P}}_{1}^{(1)}, \overline{\boldsymbol{P}}_{2}^{(2)}, \overline{\boldsymbol{P}}_{3}^{(3)}\right\}$, i.e., in $(2), \overline{\boldsymbol{P}}_{m}^{(n)}=\mathbf{0}$ for all $n \neq m$.

From (1), the decision variables of the $K$ MTs in Cell $i$ are obtained by carrying out the despreading operation, yielding

$$
\begin{aligned}
\boldsymbol{r}_{i} & =\left(\boldsymbol{C}_{i}^{f}\right)^{T}\left(\tilde{\boldsymbol{C}}_{i}^{t}\right)^{T} \boldsymbol{y}_{i} \\
& =\left(\boldsymbol{C}_{i}^{f}\right)^{T}\left(\tilde{\boldsymbol{C}}_{i}^{t}\right)^{T} \sum_{m=1}^{3} \sum_{n=1}^{3} \boldsymbol{A}_{i m} \overline{\boldsymbol{P}}_{m}^{(n)} \boldsymbol{x}_{m}+\hat{\boldsymbol{n}}_{i}, i=1,2,3
\end{aligned}
$$

where $\boldsymbol{C}_{i}^{f}=\operatorname{diag}\left\{\boldsymbol{c}_{i, 1}^{f}, \ldots, \boldsymbol{c}_{i, K}^{f}\right\}$ is a $\left(N_{f} K \times K\right)$ matrix formed by the F-domain spreading sequences for the $K$ MTs in Cell $i$. Here, $\boldsymbol{c}_{i, k}^{f}=\left[c_{i, k}^{f}[0], \ldots, c_{i, k}^{f}\left[N_{f}-1\right]\right]^{T}$, which is the F-domain spreading sequence assigned to the $k$ th MT in Cell $i . \tilde{\boldsymbol{C}}_{i}^{t}=$ $\operatorname{diag}\left\{\boldsymbol{C}_{i, 1}^{t}, \ldots, \boldsymbol{C}_{i, K}^{t}\right\}$ is a $\left(N_{t} N_{f} K \times N_{f} K\right)$ matrix formed by the T-domain spreading sequences of the $K$ MTs in Cell $i . C_{i, k}^{t}=\boldsymbol{I}_{N_{f}} \otimes$ $\boldsymbol{c}_{i, k}^{t}$ is a $\left(N_{t} N_{f} \times N_{f}\right)$ matrix, where $\boldsymbol{c}_{i, k}^{t}=\left[c_{i, k}^{t}[0], \ldots, c_{i, k}^{t}\left[N_{t}-\right.\right.$ 1] $]^{T}$, which is the T-domain spreading sequence assigned to the $k$ th MT in Cell $i$. Note that, we assume that $\left\|\boldsymbol{c}_{i, k}^{f}\right\|^{2}=\left\|\boldsymbol{c}_{i, k}^{t}\right\|^{2}=1$. Finally, in (3), we have $\hat{\boldsymbol{n}}_{i}=\left(\boldsymbol{C}_{i}^{f}\right)^{T}\left(\tilde{\boldsymbol{C}}_{i}^{t}\right)^{T} \boldsymbol{n}_{i}$.

\section{Preprocessing SCHEMES For The System}

In this section, the preprocessing matrices for the above-mentioned three types of preprocessing schemes are derived. Let us first consider the SMMSE-MUTP1.

\section{A. SMMSE-MUTP1: Single-Cell MMSE-MUTP without InterCI Sup- pression}

When the SMMSE-MUTP1 scheme is employed, each BS is assumed to have the CSI of the intracell MTs, in addition to their spreading sequences. Specifically, BS $i$ has the CSI of $\boldsymbol{A}_{i i}$ as well as the 
spreading matrices $\boldsymbol{C}_{i}^{f}$ and $\tilde{C}_{i}^{t}$. As mentioned previously, the preprocessing matrix of (2) can be written as $\overline{\boldsymbol{P}}=\operatorname{diag}\left\{\overline{\boldsymbol{P}}_{1}^{(1)}, \overline{\boldsymbol{P}}_{2}^{(2)}, \overline{\boldsymbol{P}}_{3}^{(3)}\right\}=$ $\operatorname{diag}\left\{\overline{\boldsymbol{P}}_{1}, \overline{\boldsymbol{P}}_{2}, \overline{\boldsymbol{P}}_{3}\right\}$. In this case, the decision variable vector of (3) becomes

$$
\begin{aligned}
\boldsymbol{r}_{i}= & \left(\boldsymbol{C}_{i}^{f}\right)^{T}\left(\tilde{\boldsymbol{C}}_{i}^{t}\right)^{T} \boldsymbol{A}_{i i} \overline{\boldsymbol{P}}_{i} \boldsymbol{x}_{i} \\
& +\underbrace{\sum_{m \neq i}^{3}\left(\boldsymbol{C}_{i}^{f}\right)^{T}\left(\tilde{\boldsymbol{C}}_{i}^{t}\right)^{T} \boldsymbol{A}_{i m} \overline{\boldsymbol{P}}_{m} \boldsymbol{x}_{m}}_{\text {InterCI }}+\hat{\boldsymbol{n}}_{i}
\end{aligned}
$$

After some arrangement, (4) can also be represented as

$$
\begin{aligned}
\boldsymbol{r}_{i} & =\left(\boldsymbol{C}_{i}^{t} \tilde{\boldsymbol{A}}_{i i} \boldsymbol{C}_{i}^{f}\right)^{T} \overline{\boldsymbol{P}}_{i} \boldsymbol{x}_{i}+\sum_{m \neq i}^{3}\left(\boldsymbol{C}_{i}^{t} \tilde{\boldsymbol{A}}_{i m} \boldsymbol{C}_{i}^{f}\right)^{T} \overline{\boldsymbol{P}}_{m} \boldsymbol{x}_{m}+\hat{\boldsymbol{n}}_{i} \\
& =\left(\boldsymbol{H}_{i i}^{(i)}\right)^{T} \overline{\boldsymbol{P}}_{i} \boldsymbol{x}_{i}+\sum_{m \neq i}^{3}\left(\boldsymbol{H}_{i m}^{(i)}\right)^{T} \overline{\boldsymbol{P}}_{m} \boldsymbol{x}_{m}+\hat{\boldsymbol{n}}_{i}
\end{aligned}
$$

where $\boldsymbol{C}_{i}^{t}=\left[\boldsymbol{C}_{i, 1}^{t}, \ldots, \boldsymbol{C}_{i, K}^{t}\right]$ is a $\left(N_{t} N_{f} \times N_{f} K\right)$ matrix, where $\boldsymbol{C}_{i, 1}^{t}$ was defined associated with (3), $\tilde{A}_{i m}=\operatorname{diag}\left\{\tilde{\boldsymbol{A}}_{i m, 1}, \ldots, \tilde{\boldsymbol{A}}_{i m, K}\right\}$ is a $\left(N_{f} K \times N_{f} K\right)$ matrix, where $\tilde{A}_{i m, k}$ is a $\left(N_{f} \times N_{f}\right)$ matrix defined previously. Additionally, for simplicity of description, in (5), we defined $\boldsymbol{H}_{i m}^{(i)}=\boldsymbol{C}_{i}^{t} \tilde{\boldsymbol{A}}_{i m} \boldsymbol{C}_{i}^{f}$.

Then, when the MMSE criterion is applied, the preprocessing matrix $\bar{P}_{i}$ can be obtained by solving the optimization problem [13]

$$
\overline{\boldsymbol{P}}_{i}=\arg \min _{E\left[\left\|\overline{\boldsymbol{P}}_{i} \boldsymbol{x}_{i}\right\|^{2}\right]=E\left[\left\|\boldsymbol{x}_{i}\right\|^{2}\right]}\left\{E\left[\left\|\Delta_{i}=\boldsymbol{x}_{i}-\boldsymbol{r}_{i}\right\|^{2}\right]\right\}
$$

where $E\left[\left\|\overline{\boldsymbol{P}}_{i} \boldsymbol{x}_{i}\right\|^{2}\right]=E\left[\left\|\boldsymbol{x}_{i}\right\|^{2}\right]$ is the power constraint, which satisfies $\operatorname{Tr}\left(\overline{\boldsymbol{P}}_{i}^{H} \overline{\boldsymbol{P}}_{i}\right)=K$, where $\operatorname{Tr}(\cdot)$ represents the trace operation. In (6), the estimation error vector $\Delta_{i}$ is given by

$$
\Delta_{i}=\boldsymbol{x}_{i}-\left(\boldsymbol{H}_{i i}^{(i)}\right)^{T} \overline{\boldsymbol{P}}_{i} \boldsymbol{x}_{i}-\sum_{m \neq i}^{3}\left(\boldsymbol{H}_{i m}^{(i)}\right)^{T} \overline{\boldsymbol{P}}_{m} \boldsymbol{x}_{m}-\hat{\boldsymbol{n}}_{i}
$$

Let $\boldsymbol{R}_{\triangle_{i}}$ denote the covariance matrix of the estimation error vector $\triangle_{i}$. Then, we have

$$
\begin{aligned}
\operatorname{Tr}\left(\boldsymbol{R}_{\Delta_{i}}\right)= & \operatorname{Tr}\left(\boldsymbol{I}_{K}-\left(\boldsymbol{H}_{i i}^{(i)}\right)^{T} \overline{\boldsymbol{P}}_{i}-\overline{\boldsymbol{P}}_{i}^{H}\left(\boldsymbol{H}_{i i}^{(i)}\right)^{*}\right) \\
& +\operatorname{Tr}\left(\left(\boldsymbol{H}_{i i}^{(i)}\right)^{T} \overline{\boldsymbol{P}}_{i} \overline{\boldsymbol{P}}_{i}^{H}\left(\boldsymbol{H}_{i i}^{(i)}\right)^{*}\right) \\
& +\operatorname{Tr}\left(\sum_{m \neq i}^{3}\left(\boldsymbol{H}_{i m}^{(i)}\right)^{T} \overline{\boldsymbol{P}}_{m} \overline{\boldsymbol{P}}_{m}^{H}\left(\boldsymbol{H}_{i m}^{(i)}\right)^{*}\right) \\
& +2 \sigma_{d}^{2} \operatorname{Tr}\left(\overline{\boldsymbol{P}}_{i}^{H} \overline{\boldsymbol{P}}_{i}\right)
\end{aligned}
$$

where $\operatorname{Tr}\left(\overline{\boldsymbol{P}}_{i}^{H} \overline{\boldsymbol{P}}_{i}\right)=\operatorname{Tr}\left(\boldsymbol{I}_{K}\right)$ is applied [16] and $(\cdot)^{*}$ represents the conjugate operation.

Upon taking the derivative of $\operatorname{Tr}\left(\boldsymbol{R}_{\triangle_{i}}\right)$ with respect to $\overline{\boldsymbol{P}}_{i}^{*}$ and setting the result to zero, we obtain

$$
\begin{aligned}
\overline{\boldsymbol{P}}_{i} & =\left[\left(\boldsymbol{H}_{i i}^{(i)}\right)^{*}\left(\boldsymbol{H}_{i i}^{(i)}\right)^{T}+\rho 2 \sigma_{d}^{2} \boldsymbol{I}_{N_{t} N_{f}}\right]^{-1}\left(\boldsymbol{H}_{i i}^{(i)}\right)^{*} \boldsymbol{\beta}_{i} \\
& =\tilde{\boldsymbol{P}}_{i} \boldsymbol{\beta}_{i}, i=1,2,3
\end{aligned}
$$

where $\rho$ is referred to as the noise suppression factor $[13,17]$ introduced to control the level of noise suppression or take into account of the estimation error of $\sigma_{d}^{2}$. In (9), $\boldsymbol{\beta}_{i}$ is a $(K \times K)$ diagonal matrix for power normalization satisfying $E\left[\left\|\boldsymbol{x}_{i}\right\|^{2}\right]=E\left[\left\|\overline{\boldsymbol{P}}_{i} \boldsymbol{x}_{i}\right\|^{2}\right]$. Note that, as shown in $[13,16],(9)$ can also be converted to

$$
\begin{aligned}
\overline{\boldsymbol{P}}_{i}= & \left(\boldsymbol{H}_{i i}^{(i)}\right)^{*}\left[\left(\boldsymbol{H}_{i i}^{(i)}\right)^{T}\left(\boldsymbol{H}_{i i}^{(i)}\right)^{*}+\rho 2 \sigma_{d}^{2} \boldsymbol{I}_{K}\right]^{-1} \boldsymbol{\beta}_{i}, \\
& i=1,2,3
\end{aligned}
$$

which reduces the computation complexity, when $K<N_{t} N_{f}$.

In this paper, we consider two types of power normalization [13], the first is the individual power normalization (IPN) and the second is the joint power normalization (JPN). As the name suggests, the IPN independently normalizes the transmission power of every MT after the preprocessing without considering the other MTs'. As the result, the $(k, k)$ th element in $\boldsymbol{\beta}_{i}$ is set as

$$
\boldsymbol{\beta}_{i}(k, k)=\left(\left\|\tilde{\boldsymbol{p}}_{i, k}\right\|^{2}\right)^{-1 / 2}, k=1,2, \ldots, K
$$

where $\tilde{\boldsymbol{p}}_{i, k}$ is the $k$ th column of $\tilde{\boldsymbol{P}}_{i}$. By contrast, when the JPN is employed, all the $K$ intracell MTs are considered at the same time and the normalization matrix can be expressed as

$$
\boldsymbol{\beta}_{i}=\sqrt{\frac{K}{\operatorname{Tr}\left(\tilde{\boldsymbol{P}}_{i} \tilde{\boldsymbol{P}}_{i}^{H}\right)}} \times \boldsymbol{I}_{K}
$$

B. SMMSE-MUTP2: Single-Cell MMSE-MUTP with InterCI Suppression

The proposed SMMSE-MUTP2 aims to mitigate the InterCI in addition to suppressing the IntraCI. For this objective, according to [17], the transmitter preprocessing matrix can be obtained from a corresponding MUD problem. Specifically, when the multicell scenario is considered, the uplink observation equation equivalent to the downlink observation equation of (5) is given by

$$
\boldsymbol{y}_{i}^{(u)}=\sum_{m=1}^{3} \boldsymbol{H}_{i m}^{(i)} \boldsymbol{x}_{m}+\boldsymbol{n}_{i}^{(u)}, i=1,2,3
$$

where $\boldsymbol{x}_{m}$ collects the symbols transmitted by the $K$ MTs in Cell $m$, $\boldsymbol{H}_{i m}^{(i)}$ is a $\left(N_{t} N_{f} \times K\right)$ matrix defined in (5), and $\boldsymbol{n}_{i}^{(u)}$ is a $N_{t} N_{f}$ length Gaussian noise vector, each element of which has a zero mean and a variance of $2 \sigma_{u}^{2}$. Then, when the MMSE-MUD is employed, the MUD weight matrix used by BS $i$ can be expressed as [13]

$$
\begin{aligned}
\boldsymbol{W}_{i} & =\boldsymbol{R}_{\boldsymbol{y}_{i}^{(u)}}^{-1} \boldsymbol{H}_{i i}^{(i)} \\
& =\left(\sum_{m=1}^{3} \boldsymbol{H}_{i m}^{(i)}\left(\boldsymbol{H}_{i m}^{(i)}\right)^{H}+2 \sigma_{u}^{2} \boldsymbol{I}_{N_{t} N_{f}}\right)^{-1} \boldsymbol{H}_{i i}^{(i)}
\end{aligned}
$$

Correspondingly, according to [17], the preprocessing matrix in MMSE sense can be obtained via its relationship with the MMSEMUD, which can be expressed as as

$$
\begin{aligned}
\overline{\boldsymbol{P}}_{i}= & \left.\boldsymbol{W}_{i}^{*} \boldsymbol{\beta}_{i}\right|_{\rho \sigma_{d}^{2} \rightarrow \sigma_{u}^{2}} \\
= & {\left[\sum_{n=1}^{3}\left(\boldsymbol{H}_{i m}^{(i)}\right)^{*}\left(\boldsymbol{H}_{i m}^{(i)}\right)^{T}+\rho 2 \sigma_{d}^{2} \boldsymbol{I}_{N_{t} N_{f}}\right]^{-1}\left(\boldsymbol{H}_{i i}^{(i)}\right)^{*} \boldsymbol{\beta}_{i} } \\
& \quad i=1,2,3
\end{aligned}
$$

where $a \rightarrow b$ means using $a$ to replace $b$.

Note that, in (14), the autocorrelation matrix $\boldsymbol{R}_{\boldsymbol{y}_{i}^{(u)}}$ can be directly estimated from the uplink without requiring to know the uplink channels of the $3 K$ MTs. Hence, the preprocessing scheme of SMMSEMUTP2 is relatively easy to implement in practice. Furthermore, if the noise variances of the uplink and downlink are similar, MMSE-MUTP is achieved. In the cases where the uplink and downlink noise variances are not the same, once $\boldsymbol{R}_{\boldsymbol{y}_{i}^{(u)}}$ is estimated, the values of it diagonal elements may be modified, in order to attain improved performance. This is reflected by the noise suppression factor $\rho$ seen in (15). 
Finally, as described in Section III-A, the power normalization for the SMMSE-MUTP2 can be based on the IPN or JPN. The corresponding result for $\boldsymbol{\beta}_{i}$ in (15) is given by (10) or (11).

\section{CMMSE-MUTP: Multi-Cell Cooperative MMSE-MUTP}

When the CMMSE-MUTP is considered, as the three BSs ideally cooperate with each other, we assume that there is a virtual central signal processing unit, which is connected with the three BSs with unlimited backhaul links. Hence, all the CSI as well as the data to be transmitted can be used for implementing the preprocessing. In this case, the MMC/DS-CDMA system can be viewed as a virtual singlecell system. Consequently, the MUTP for the three cells can be jointly designed.

Let $\boldsymbol{r}=\left[\boldsymbol{r}_{1}^{T}, \boldsymbol{r}_{2}^{T}, \boldsymbol{r}_{3}^{T}\right]^{T}$, where $\boldsymbol{r}_{i}$ is given by (3). Then, it can be shown that $\boldsymbol{r}$ can be expressed as

$$
\begin{aligned}
\boldsymbol{r} & =\left(\boldsymbol{C}^{t} \tilde{\boldsymbol{A}} \boldsymbol{C}^{f}\right)^{T} \overline{\boldsymbol{P}} \boldsymbol{x}+\boldsymbol{n} \\
& =\boldsymbol{H}^{T} \overline{\boldsymbol{P}} \boldsymbol{x}+\boldsymbol{n}
\end{aligned}
$$

where $\boldsymbol{r}$ is a $3 K$-length vector, $\boldsymbol{C}^{t}=\operatorname{diag}\left\{\boldsymbol{C}_{1}^{t}, \boldsymbol{C}_{2}^{t}, \boldsymbol{C}_{3}^{t}\right\}$ is the $\left(3 N_{t} N_{f} \times 3 N_{f} K\right)$ T-domain spreading matrix, and $\boldsymbol{C}^{f}=$ $\operatorname{diag}\left\{\boldsymbol{C}_{1}^{f}, \boldsymbol{C}_{2}^{f}, \boldsymbol{C}_{3}^{f}\right\}$ is the $\left(3 N_{f} K \times 3 K\right)$ F-domain spreading matrix, where both $\boldsymbol{C}_{i}^{t}$ and $\boldsymbol{C}_{i}^{f}$ were defined in Section II. In (16), the matrix $\tilde{A}$ related to the downlink channels is given by

$$
\tilde{A}=\left[\begin{array}{ccc}
\tilde{A}_{11} & \tilde{A}_{21} & \tilde{A}_{31} \\
\tilde{A}_{12} & \tilde{\boldsymbol{A}}_{22} & \tilde{\boldsymbol{A}}_{32} \\
\tilde{A}_{13} & \tilde{A}_{23} & \tilde{A}_{33}
\end{array}\right]
$$

where $\tilde{A}_{i m}$ was discussed in Section III-A, which is a $\left(N_{f} K \times N_{f} K\right)$ matrix. Therefore, $\tilde{A}$ is a $\left(3 N_{f} K \times 3 N_{f} K\right)$ matrix. Furthermore, for convenience, in (16), we defined $\boldsymbol{H}=\boldsymbol{C}^{t} \tilde{\boldsymbol{A}} \boldsymbol{C}^{f}$, which is a $\left(3 N_{t} N_{f} \times\right.$ $3 K$ ) equivalent channel matrix.

Based on (16), the preprocessing matrix $\bar{P}$ in the principles of MMSE-MUTP can be derived by following the steps for the SMMSEMUTP1, as described in Section III-A. The resultant preprocessing matrix can be expressed as

$$
\begin{aligned}
\overline{\boldsymbol{P}} & =\left(\boldsymbol{H}^{*} \boldsymbol{H}^{T}+\rho 2 \sigma_{d}^{2} \boldsymbol{I}_{3 N_{t} N_{f}}\right)^{-1} \boldsymbol{H}^{*} \boldsymbol{\beta} \\
& =\tilde{\boldsymbol{P}} \boldsymbol{\beta}
\end{aligned}
$$

where the power normalization matrix is $\beta=\operatorname{diag}\left\{\boldsymbol{\beta}_{1}, \boldsymbol{\beta}_{2}, \boldsymbol{\beta}_{3}\right\}$ with $\boldsymbol{\beta}_{m}=\operatorname{diag}\left\{\beta_{m 1}, \ldots, \beta_{m K}\right\}$. For the sake of comparison, three types of power normalization techniques are considered for the CMMSEMUTP, which are the IPN, single-cell joint power normalization (SJPN) and the multi-cell joint power normalization (MJPN). The IPN and SJPN are the same as that considered in Section III-A, which were given in (11) and (12), respectively. By contrast, when the MJPN is employed. The normalization matrix is given by

$$
\boldsymbol{\beta}=\sqrt{\frac{3 K}{\operatorname{Tr}\left(\tilde{\boldsymbol{P}} \tilde{\boldsymbol{P}}^{H}\right)}} \times \boldsymbol{I}_{3 K}
$$

Let us now provide a range of performance results to characterize the preprocessing schemes.

\section{Performance Results}

In this section, we provide a range of simulation results for demonstrating the achievable error performance of the MMC/DS-CDMA systems employing various MUTP schemes considered. For all the figures shown, we assume BPSK modulation and frequency selective Rayleigh fading channels. Furthermore, we assume ideal noise variance estimation, setting the noise suppression factor $\rho=1$.

Figs. 2 and 3 show the BER performance of the MMC/DS-CDMA systems employing different MUTP schemes, when different number

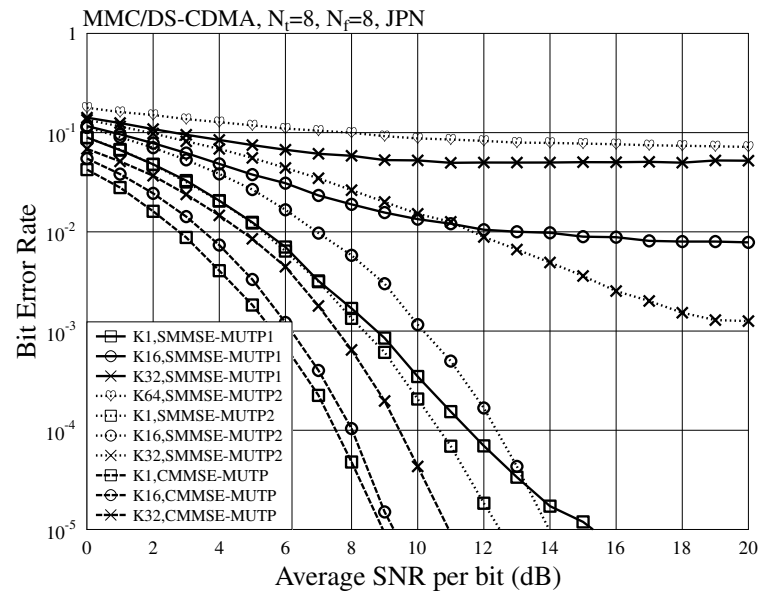

Fig. 2. BER performance of MMC/DS-CDMA system with various of MUTP schemes over frequency selective Rayleigh fading channels, where $N_{t}=8$, $N_{f}=8$, and joint power normalization is employed.

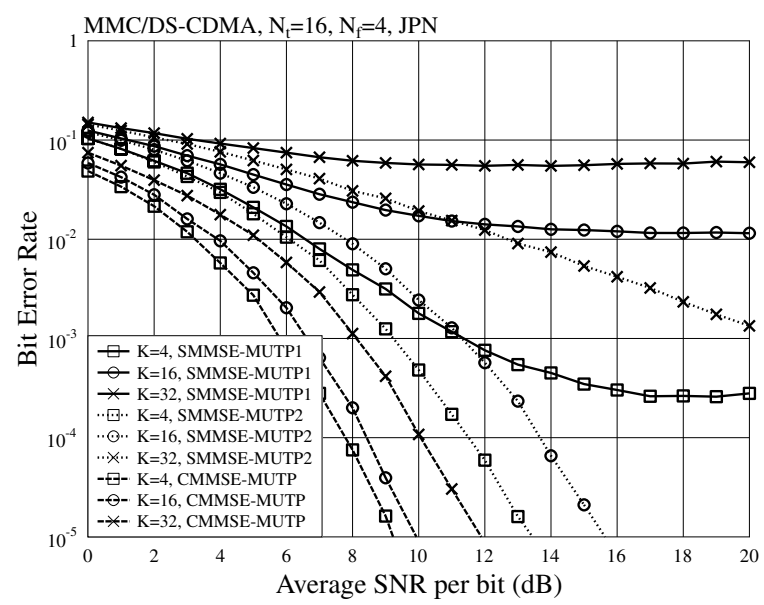

Fig. 3. BER performance of MMC/DS-CDMA system with various of MUTP schemes over frequency selective Rayleigh fading channels, where $N_{t}=16$, $N_{f}=4$, and joint power normalization is employed.

of MTs per cell are supported. The differences between the parameters used in Fig. 2 and Fig. 3 are that $N_{t}=N_{f}=8$ for Fig. 2 and $N_{t}=16, N_{f}=4$ for Fig. 3, while the total spreading factor remains $N_{t} N_{f}=64$. From the two figures, we may have the following observations. First, among the three MUTP schemes, the CMMSEMUTP always achieves the best BER performance, owing to the BS cooperation, which yields antenna diversity or macro-diversity. However, we should remember that the CMMSE-MUTP also demands the highest complexity among the three MUTP schemes considered. Second, for a given MUTP scheme, the BER increases as the number of MTs increases, which is caused by the increased amount of InterCI as well as the IntraCI. Third, owing to the capability of InterCI suppression, the SMMSE-MUTP2 outperforms the SMMSE-MUTP1. From the figures, error floors are observed for the SMMSE-MUTP1 in the context of all the cases. For the SMMSE-MUTP2, error floors also present, when the system is full-load, i.e., when $K=64$. This is because, in this case, the interference, including InterCI and IntraCI, is beyond the capability of the SMMSE-MUTP2. Finally, when comparing the results in Fig. 2 with the corresponding results in Fig. 3, we can find that, for a given MUTP supporting the same number of MTs, the BER performance in Fig. 2 is better than that in 


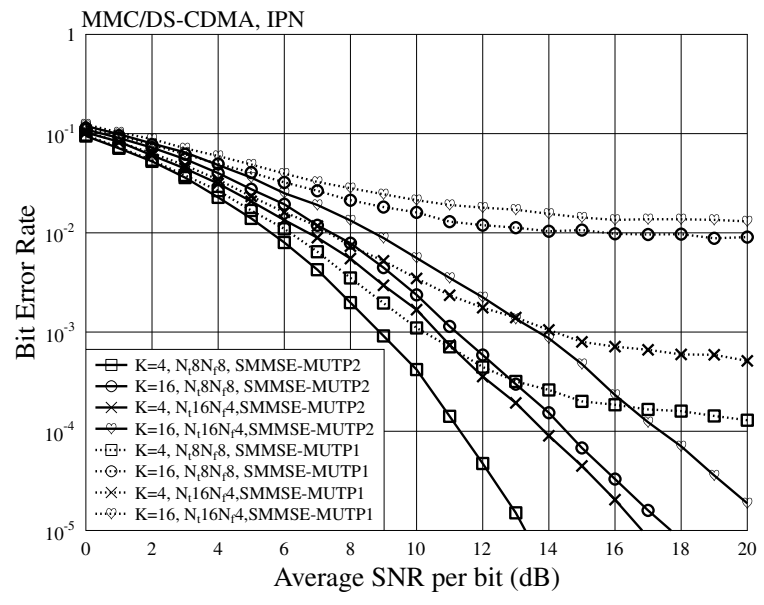

Fig. 4. BER performance of MMC/DS-CDMA systems with various MUTP schemes employing individual power normalization, when communicating over frequency selective Rayleigh fading channels.

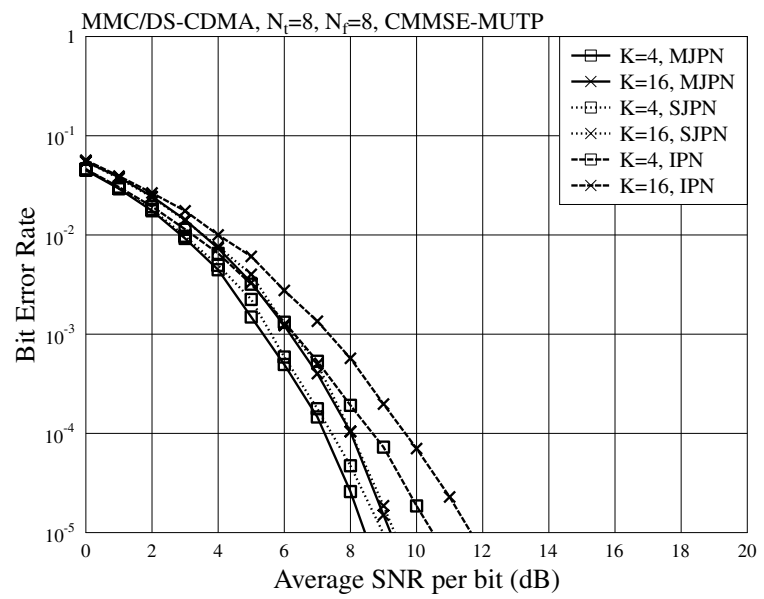

Fig. 5. BER performance of MMC/DS-CDMA systems with the CMMSEMUTP scheme employing various power normalization schemes, when communicating over frequency selective Rayleigh fading channels.

Fig. 3. This is because, in Fig. 2, using $N_{f}=8$ subcarriers generates a higher frequency diversity gain than using $N_{f}=4$ subcarriers in Fig. 3.

Fig. 4 investigates the BER performance of the MMC/DS-CDMA systems employing respectively the SMMSE-MUTP1 and SMMSEMUTP2, when the IPN is applied. In comparison with the scenarios considered in Figs. 2 and 3, where the JPN is applied, the BER performance of a corresponding scheme shown in Fig. 4 becomes worse. Furthermore, Fig. 4 shows that, for a given scheme and a given number of MTs supported, the SMMSE-MUTP2 outperforms the SMMSE-MUTP1, although both of them have a similar complexity.

Finally, in Fig. 5, we study the impact of the three power normalization schemes, namely, the IPN, SJPN and MJPN, for the CMMSEMUTP. Explicitly, the MJPN outperforms the SJPN, while the scheme employing the IPN is the worst among the three schemes considered. Additionally, as seen in Fig. 5, the advantage of MJPN over SJPN vanishes, as the number of MTs supported is increased from $K=4$ to $K=16$.

\section{Conclusions}

In this contribution, we have studied the MUTP in the downlink MMC/DS-CDMA systems employing both T- and F-domain spread- ing. Three types of MUTP schemes, namely, the SMMSE-MUTP1, SMMSE-MUTP2 and the CMMSE-MUTP, as well as the powerallocation in these schemes have been investigated. Among the three MUTP schemes, explicitly, the CMMSE-MUTP demands significantly higher complexity than the SMMSE-MUTP1 and SMMSE-MUTP2, while the SMMSE-MUTP1 and SMMSE-MUTP2 have a similar complexity. The performance results show that the CMMSE-MUTP is capable of achieving the best error performance among the three MUTP schemes. The SMMSE-MUTP2 can effectively mitigate both the IntraCI and the InterCI, and achieve promising error performance that is much better than the SMMSE-MUTP1, especially, when the number of MTs supported by each cell is not very high. Considering that the SMMSE-MUTP2 has a similar complexity as the SMMSEMUTP1, the SMMSE-MUTP2 may constitute a promising MUTP scheme for the MMC/DS-CDMA systems, where information exchange among BSs is impractical.

\section{REFERENCES}

[1] F. Rashid-Farrokhi, K.J.R. Liu and L. Tassiulas, "Transmit beamforming and power control for cellular wireless systems," IEEE Journal on Selected Areas in Communications, vol. 16, no. 8, pp. 1437-1450, Oct 1998.

[2] F. Rashid-Farrokhi, L. Tassiulas and K.J.R. Liu, "Joint optimal power control and beamforming in wireless networks using antenna arrays," IEEE Transactions on Communications, vol. 46, no. 10, pp. 1313-1324, Oct 1998.

[3] M. Schubert and H. Boche, "Solution of the multiuser downlink beamforming problem with individual SINR constraints," IEEE Transactions on Vehicular Technology, vol. 53, no. 1, pp. 18-28, Jan 2004.

[4] W. Yu and T. Lan, "Transmitter Optimization for the Multi-Antenna Downlink with Per-antenna Power Constraints," IEEE Transactions on Signal Processing, vol. 55, no. 6, pp. 2646-2660, June 2007.

[5] M.K. Karakayali, G.J. Foschini and R.A. Valenzuela, "Network coordination for spectrally efficient communications in cellular systems," IEEE Transactions on Wireless Communications, vol. 13, no. 4, pp. 56-61, Aug 2006.

[6] W. Choi and J.G. Andrews, "Downlink performance and capacity of distributed antenna systems in a multicell environment," IEEE Transactions on Wireless Communications, vol. 6, no. 1, pp. 69-73, Jan 2007.

[7] A. Tajer, N. Prasad and X.D. Wang, "Robust linear precoder design for multi-cell downlink transmission," IEEE Transactions on Signal Processing, vol. 59, no. 1, pp. 235-251, Jan 2011.

[8] R. Zhang and L. Hanzo, "Cooperative downlink multicell preprocessing relying on reduced-rate back-haul date exchange," IEEE Transactions on Vehicular Technology, vol. 60, no. 2, pp. 539-545, Feb 2011.

[9] D. Ponukumati, F. Gao, M. Bode and and X. Liao, "Multicell downlink beamforming with imperfect channel knowledge at both transceiver sides," IEEE Communications Letters, vol. 15, no. 10, pp. 1075-1077, Oct 2011.

[10] S. Shamai, O. Somekh and B. M. Zaidel, "Multi-cell communications: An information theoretic perspective," Joint Workshop on Communications and Coding, Florence, Italy, Oct 2004.

[11] O. Somekh, B. M. Zaidel and S. Shamai, "Sum rate characterization of joint multiple cell-site processing," IEEE Transactions on Information Theory, vol. 53, no. 12, pp. 4473-4497, Dec 2007.

[12] J. Zhang and J. G. Andrews, "Adaptive spatial intercell interference cancellation in multicell wireless networks," IEEE Journal on Selected Areas in Communications, vol. 28, no. 9, pp. 1455-1468, Dec 2010.

[13] L.-L. Yang, "Multicarrier Communications" John Wiley \& Sons, Chichester, U.K., 2009.

[14] P. Pan, L.-L. Yang and Y. Zhang, "Spectral-efficiency of time-frequencydomain spread multicarrier DS-CDMA in frequency-selective Nakagamim fading channels," in IEEE VTC2008-Fall, pp. 1-5, Sep 2008.

[15] P. Pan, L.-L. Yang and Y. Zhang, "Time-frequency iterative multiuser detection in time-frequency-domain spread multicarrier DS-CDMA systems over Nakagami- $m$ fading channels," European Transactions on Telecommunications, vol. 22, no. 1, pp. 2-13, 2011. [Online]. Available: http://dx.doi.org/10.1002/ett.1439

[16] R. L.-U. Choi and R. D. Murch, "New transmit schemes and simplified receivers for MIMO wireless communication systems", IEEE Transactions on Wireless Communications, Vol. 2, No. 6, pp. 1217 - 1230, Nov. 2003.

[17] L.-L. Yang, "Design linear multiuser transmitters from linear multiuser receivers," IEEE International Conference on ICC, pp. 5258 -5263, June 2007. 